\title{
Realized Volatility of Precious Metal Returns: HAR-RV
}

\author{
Roengchai Tansuchat ${ }^{1,{ }^{*}}$ \\ ${ }^{1}$ Faculty of Economics, Chiang Mai University \\ Chiang Mai, Thailand \\ *email: roengchaitan@gmail.com
}

\begin{abstract}
This paper applies ultra-high frequency data particularly tick-by-tick data from three precious metals, namely gold, silver and platinum to estimate realized volatility, and model HAR-RV models evaluate Value-at-Risk. The tick-by-tick data from January 1, 2011 to December 8, 2016 covering 2,070,720 of tick data per year with the total days of 1,439 days were used. The med $R V$ is used to calculate the daily realized volatility and forwardly establish and compare HAR-RV in different functional form. The empirical results show that the minimum VaR and daily capital loss of precious metal are gold and follow by silver and platinum respectively.
\end{abstract}

\section{Keywords: Realized Volatility, Precious Metal Returns, HAR-RV, Value-at-Risk}

\section{Introduction}

In financial research, volatility is played an essential role in the application of financial trading, price predicting, hedging against portfolio risk or risk measurement particularly value-at risk (VaR) not only in financial markets but also commodity market. Although, volatility of financial asset cannot be observed, it is usually applied to measure and evaluate risk or uncertainty. Therefore, volatility modeling is imperative. In financial econometrics, there are three approaches used to estimate the volatility, namely conditional volatility $(\mathrm{CV})$, stochastic volatility (SV) and realized volatility (RV). The most popular volatility modeling approach is conditional volatility whereas the estimated volatility or variance is estimated conditionally or dependently on the new information and it can be changed day by day. The most popular ways to model the conditional volatility are deterministically the GARCH-type family. In case of stochastic volatility models, the square volatilities are formulated probabilistically in term of latent variable in which is assumed to follow the first order autoregressive process through the recent development of algorithms such as Monte Carlo Markov Chain (MCMC) and quasi-maximum likelihood estimation. In practical frameworks, GARCH and SV model is normally estimated with daily or lower frequency data.

Hansen and Lunde [1] pointed that by using high frequency financial data, when the observations were made available over the short periods, it can be considerably improved volatility forecasting. McAleer and Medeiros [2] provided the review of realized volatility (RV). The realized volatility purposed by Anderson and Bollerslev [3] is a non-parametric measure and a proxy for non-observed integrated volatility. By adding up the squared intraday returns that provide the realized variance is commonly used to measure realized volatility. In order to model the realized volatility, ARFIMA and/or FIGARCH models are usually adopted (eg. Beltratti and Morana [4] and Fuertes and Olmo [5]) as well as Heterogeneous Autoregressive realized Variance (HAR-RV) models of Corsi [6]. In the empirical literatures, most of research studies applied the realized volatility and those mentioned models in stock markets (eg. Giot and Laurent [7]). However, RV is rarely applied in precious or non-precious metal to model and evaluate Value-at-Risk expected for Klein [8], made the forecasting comparison between RV and GARCH, APARCH, FIGARCH and FIAPARCH and Todorova, et al. [9] employed multivariate HAR model to evaluate the spillovers between five non-ferrous metals.

This paper proposes ultra-high frequency data particularly tick-by-tick data from three precious metals, namely gold, silver and platinum to estimate RV and HAR-RV models to evaluate Value-at-Risk. The remainder of the paper is structured as follows: Section 2 introduces the theoretical frameworks of HAR-RV model and the estimation of VaR, Section 3 describes the price 
and return series of the three precious metals, Section 4 presents the empirical results and Section 5 is to conclude the findings.

\section{Econometrics Model}

\subsection{RV}

The realized variance was purposed by Anderson et al. [2]. The log returns are transformed to daily $\mathrm{RV}$ by med $R V$ estimation (Anderson et al. [10]) in order to estimated daily $\mathrm{RV}$. The med $R V$ will select the median of three adjacent absolute returns as follows:

$$
\operatorname{med} R V_{t}=\frac{\pi}{6-4 \sqrt{3}+\pi}\left(\frac{M}{M-2}\right) \sum_{i=2}^{M-1} \operatorname{med}\left(\left|r_{t, i-1}\right|,\left|r_{t, i}\right|,\left|r_{t, i+1}\right|\right)^{2}
$$

where $r$ is a return with $i=1, \ldots, M$ in period $t$.

\subsection{Long-Memory}

The estimated daily RV from med $R V_{t}$ is checked long memory property by two long memory tests, namely R/S test (Hurst [11]) and GPH test (Geweke and Porter-Hudak [12]). The R/S test of range over standard deviation test is defined as:

$$
Q_{T}=\frac{1}{s_{T}}\left[\max _{1 \leq k \leq T} \sum_{j=1}^{k}\left(y_{j}-\bar{y}\right)-\min _{1 \leq k \leq T} \sum_{j=1}^{k}\left(y_{j}-\bar{y}\right)\right]
$$

where $\bar{y}=1 / T \sum_{i=1}^{T} y_{j}$ and $s_{T}=\sqrt{1 / T \sum_{i=1}^{T}\left(y_{j}-\bar{y}\right)}$. The null hypothesis of R/S test is no long term dependence. Alternatively, the GPH test, given the fractionally integrated process of $y$ the spectral density $f(\omega)=\left[4 \sin ^{2}\left(\frac{\omega}{2}\right)\right]^{-d} f_{u}(\omega)$ where $\omega$ is the Fourier frequency, and $f_{u}(\omega)$ is the spectral density corresponding to $u_{t}$. The $d$ can be estimated by least square of the following regression:

$$
\ln f\left(\omega_{j}\right)=\beta-d \ln \left[4 \sin ^{2}\left(\frac{\omega_{j}}{2}\right)\right]+e_{j}
$$

for $j=1,2, \ldots, n_{f}(T)$. The distribution of $\hat{d}$ with the large sample if $n_{f}(T)=T^{\alpha}, 0<\alpha<1$ is normal distribution, $d \sim N\left(d, \frac{\pi^{2}}{6 \sum_{j=1}^{n_{f}}\left(U_{j}-\bar{U}\right)^{2}}\right)$ with $U_{j}=\ln \left[4 \sin ^{2}\left(\frac{\omega_{j}}{2}\right)\right]$, and $\bar{U}$ is the sample mean of $U_{j}$, $j=1, \ldots, n_{f}$. The null hypothesis of no long memory $d=0$ is tested by traditional $t$ statistic.

\subsection{HAR}

The simple autoregressive structure of realized volatility over different interval sizes or HAR(3)-RV one day $\left(\tilde{\sigma}_{t}^{(d)}\right)$ is given as:

$$
R V_{t+1 d}^{(d)}=c+\beta^{(d)} R V_{t}^{(d)}+\beta^{(w)} R V_{t}^{(w)}+\beta^{(m)} R V_{t}^{(m)}+\omega_{t+1 d}
$$

where $R V_{t}^{(d)}, R V_{t}^{(w)}$ and $R V_{t}^{(m)}$ are the realized volatility of one day, one week (5 days) and one month (22 days), respectively, and $\omega_{t+1 d}=\tilde{\omega}_{t+1 d}^{(d)}-\omega_{t+1 d}^{(d)}$. In this paper the square root form and logarithmic form of above model are applied, so the new models are equated as follows:

$$
\begin{aligned}
\left(R V_{t+1 d}^{(d)}\right)^{1 / 2} & =c+\beta^{(d)}\left(R V_{t}^{(d)}\right)^{1 / 2}+\beta^{(w)}\left(R V_{t}^{(w)}\right)^{1 / 2}+\beta^{(m)}\left(R V_{t}^{(m)}\right)^{1 / 2}+\omega_{t+1 d} \\
\log \left(R V_{t+1 d}^{(d)}\right) & =c+\beta^{(d)} \log \left(R V_{t}^{(d)}\right)+\beta^{(w)} \log \left(R V_{t}^{(w)}\right)+\beta^{(m)} \log \left(R V_{t}^{(m)}\right)+\omega_{t+1 d}
\end{aligned}
$$

\subsection{VaR}

Value at Risk quantifies the market risk of asset to future market fluctuations (Sarma et al. [13]). This is usually defined as the amount of money that might be lost within a given horizontal time with a certain probability $\alpha$.

$$
P\left(P_{t} \leq P_{t-1}-\operatorname{VaR}_{t}(\alpha)\right)=\alpha
$$

In terms of returns, Value at Risk is usually defined as: 


$$
P\left(r_{t} \leq-\operatorname{VaR}_{t}(\alpha)\right)=\alpha
$$

Thus, the paper used VaR Variance-Covariance Method which was defined as follows:

$$
\operatorname{VaR}_{t+1}^{\alpha}=\hat{\mu}_{t+1}+\hat{\sigma}_{t+1} \cdot \text { Quantile }
$$

where $\mu_{t}$ and $\sigma_{t}$ are mean and variance, resepctively.

\section{Data}

The ultra-high frequency at one-minute frequency in the spot price of 4 precious metals, namely gold, silver, platinum and palladium traded in Chicago board of trade were collected from Thomson Reuters DataStream, from Financial Investment Center (FIC), Faculty of Economics, Chiang Mai University (CMU). The study period, started from the period of January 1, 2011 to December 8, 2016 with the total of 2,070,720 tick data per year conversed with 1,439 daily RV were used. The Fig. 1 shows the plot of one-minute frequency of three precious metal prices. The intraday prices $\left(p_{t(i)}\right)$ are calculated by continuous compounding returns and defined as $R_{t(i+1)}=\ln \left(p_{t(i+1)}\right)-\ln \left(p_{t(i)}\right)$, the daily realized volatilities were continually estimated by MedRV techniques of Anderson et al. [10] and was presented in fig. 2. The plots of price and estimated RV of each precious metal are moved absolutely in a similar manner.
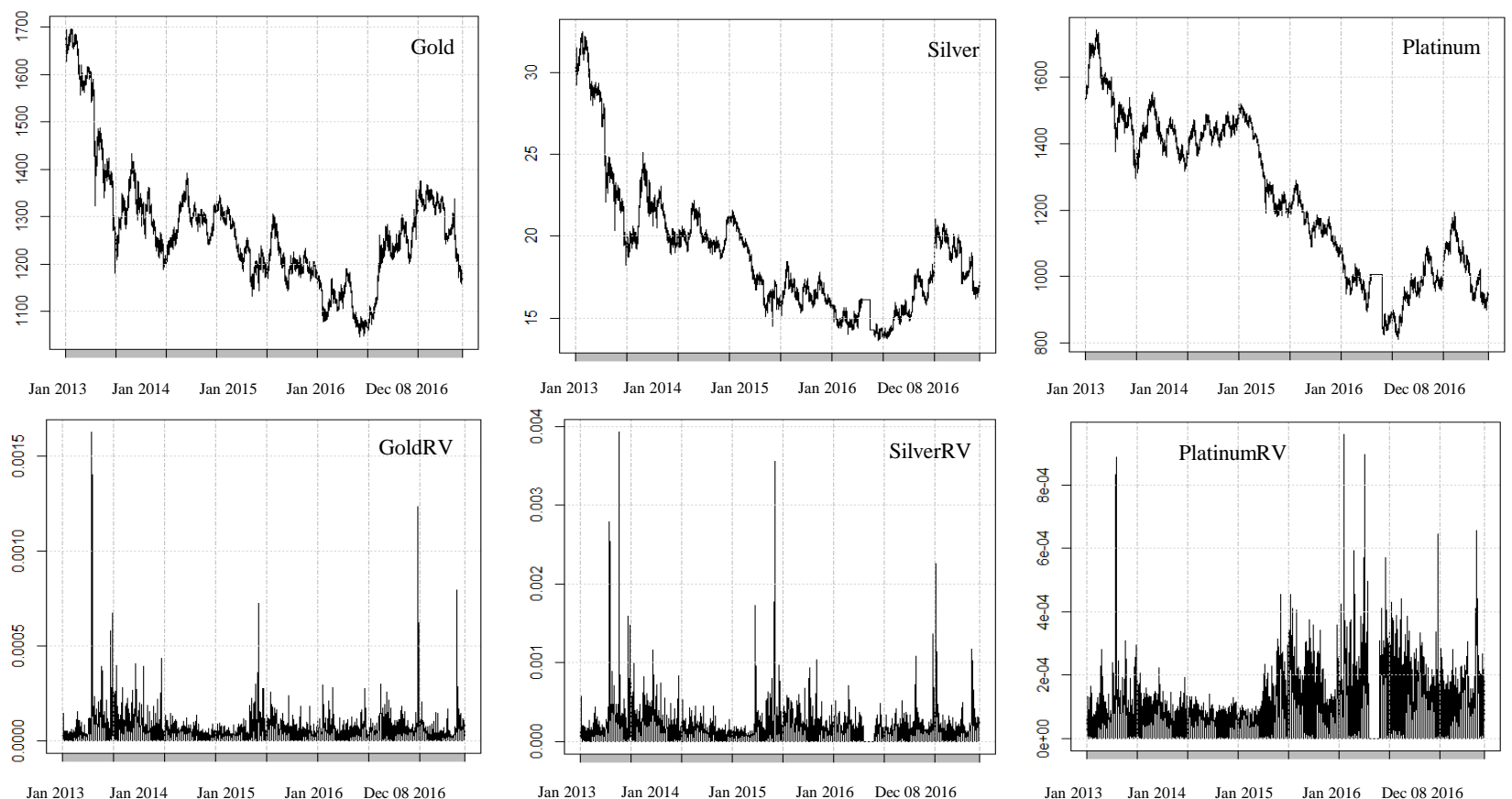

Figure 1. Intraday precious metal prices and estimated realized volatility

\section{Empirical Results}

The paper estimates VaR and daily capital loss of precious metal prices based on realized volatility and HAR model by using tick-by-tick data. Firstly, one minute data of precious price were transformed to return and continually estimated the daily realized volatily and HAR-RV model. The daily realized volatily series detected the long memery feature by using R/S and GPH test. All tests were statistically rejected the null hypothesis (Table 1). In this study, the HAR-RV is estimated in three different formats, namely normal $\mathrm{RV}$, square root $\mathrm{RV}(\sqrt{R V})$ and logarithm $\mathrm{RV}(\log (R V))$. In Table 1 illustrates the coefficients of HAR-RV model with normal RV, square root RV and logarithm RV for each precious metal. All coefficients are statistically significant at $1 \%$ level. In addition, the HAR-RV model with normal form is methodologically the best one to estimate HAR-RV model according to the minimum value of Akaike information criteria (AIC) and 
Bayesian information criterion (BIC). In term of the estimations of gold, silver and platinum equation, the daily and monthly parameter for daily effected on RV are positive whereas the weekly parameter is negative.

Table 1 HAR-RV Estimation

\begin{tabular}{|c|c|c|c|c|c|c|c|c|c|}
\hline & RV & $\sqrt{R V}$ & $\log (R V)$ & RV & $\sqrt{R V}$ & $\log (R V)$ & RV & $\sqrt{R V}$ & $\log (R V)$ \\
\hline$\beta_{0}$ & $0.00002^{* * * *}$ & $0.0018 * * *$ & $28.883 * * *$ & $0.00006^{* * *}$ & $0.0024 * * *$ & $16.227^{*}$ & $0.00002 * * *$ & $0.0019 * * *$ & $14.687 * * *$ \\
\hline$\beta_{D}$ & $0.378 * * *$ & $0.378 * * *$ & $0.489 * * *$ & $0.319 * * *$ & $0.374 * * *$ & $0.491 * * *$ & $0.465 * * *$ & $0.435^{* * *}$ & $0.381 * * *$ \\
\hline$\beta_{w}$ & $-0.156^{* * * *}$ & $-0.503 * * *$ & $-0.891 * * *$ & $-0.198 * * *$ & $-0.435^{* * *}$ & $-0.849 * * *$ & $-0.560 * * *$ & $-1.031 * * *$ & $-1.489 * * *$ \\
\hline$\beta_{M}$ & $0.442 * * *$ & $0.790 * * *$ & $1.136^{* * *}$ & $0.563 * * *$ & $0.781 * * *$ & $1.167 * * *$ & $0.908 * * *$ & $1.382 * * *$ & $1.948 * * *$ \\
\hline$R^{2}$ & 0.170 & 0.210 & 0.350 & 0.140 & 0.220 & 0.380 & 0.320 & 0.380 & 0.490 \\
\hline $\operatorname{Adj} . R^{2}$ & 0.170 & 0.210 & 0.350 & 0.140 & 0.220 & 0.380 & 0.320 & 0.380 & 0.490 \\
\hline$R / S$ & $0.706^{*}$ & $0.686^{*}$ & - & $0.708^{*}$ & $0.691^{*}$ & - & $0.709 *$ & $0.682 *$ & - \\
\hline$G P H$ & $0.342 *$ & $0.378^{*}$ & $0.632 * *$ & $0.493 * *$ & $0.496 * *$ & $0.636 * *$ & $0.373 *$ & $0.389 *$ & $0.605 * *$ \\
\hline AIC & $-22,560$ & $-11,632$ & 12,929 & $-19,607$ & $-10,064$ & 12,374 & $-22,303$ & $-11,230$ & 12,018 \\
\hline $\mathrm{BIC}$ & $-22,533$ & $-11,606$ & 12,955 & $-19,581$ & $-10,037$ & 12,401 & $-22,276$ & $-11,204$ & 12,044 \\
\hline
\end{tabular}

Note: $*$, **, and $* * *$ represent the significant at the $10 \%, 5 \%$ and $1 \%$ level, respectively.
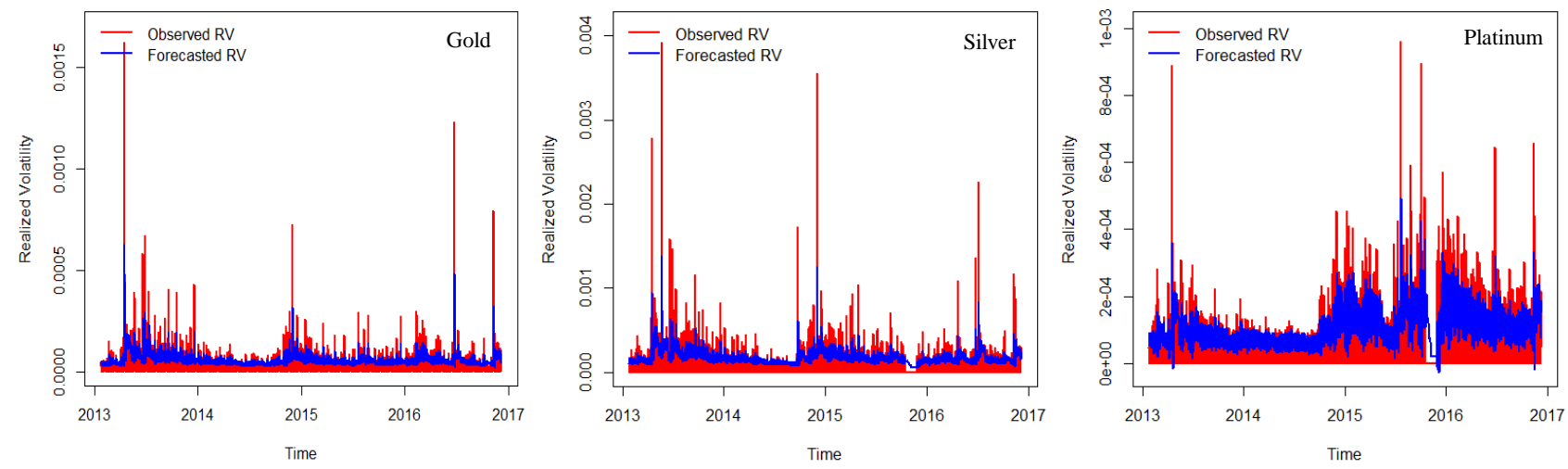

Figure 2. Daily RV forecasting from HAR-RV models

Fig. 2 presents the plot of daily RV forecasting obtained from HAR-RV model in normal form, and esitmated RV. Table 2 presents the results of Value at Risk and capital loss for $1 \%, 5 \%$ and $10 \%$. The minium VaR is Platinum and followed by gold and sliver, respectively. If the estimation of VaR of gold at $1 \%$ confidence level for 1 trading day is increased by $1.31 \%$, meaning that the worst daily loss will not expectially exceed by $1.31 \%$. For example, if we invest $\$ 1,000,000$ with worst daily loss, it will not exceed $\$ 13,175.81$.

Table 2.Value at Risk and Capital Loss

\begin{tabular}{lcccccc}
\hline & \multicolumn{3}{c}{ VaR } & & \multicolumn{3}{c}{ Capital Loss* } \\
\cline { 2 - 7 } & Gold & Sliver & Platinum & Gold & Sliver & Platinum \\
\hline $\mathbf{9 9 \%}$ & 0.0131 & 0.0364 & 0.0101 & $13,175.81$ & $36,444.85$ & $10,091.54$ \\
$\mathbf{9 5 \%}$ & 0.0074 & 0.0202 & 0.0038 & $7,478.29$ & $20,284.66$ & $3,817.60$ \\
$\mathbf{9 0 \%}$ & 0.0043 & 0.0114 & 0.0004 & $4,378.16$ & $11,491.62$ & 403.83 \\
\hline
\end{tabular}




\section{Conclusions}

This paper evaluates Value-at-Risk and capital loss of precious metals spot prices traded on the London Metal Exchange by using realized volatility techniques and HAR-RV models. The tick-by-tick data started from January 1, 2011 to December 8, 2016 covering 2,070,720 of tick data per year and totally 1,439 days were used. The med $R V$ is used to calculate the daily realized volatility and continually establish and compare HAR-RV models in different functional form. The empirical results show that the best model is HAR-RV in normal form and the minimum VaR and daily capital loss of precious metal is platinum and followed by gold and slivers, respectively.

\section{Acknowledgement}

The author would like to thank to Faculty of Economics, Chiang Mai University (CMU) for the financial support, and the collaborating researchers, research assistants for giving their generous time and expertise.

\section{References}

[1] P.R. Hansen, and A. Lunde: Forecasting Volatility using High-Frequency Data, edited by M. P. Clements and D. F. Hendry, The Oxford Handbook of Economic Forecasting (2011). p. 525-556.

[2] M. McAleer and M.C. Dedeiros: Realized Volatility: A Reivew. Journal Econometric Reviews 27 (2008), p. 10-45.

[3] T.G. Andersen, T. Bollerslev, F. X. Diebold, and P. Labys: Modeling and Forecasting Realized Volatility, Econometrica 71 (2003), p. 579-625.

[4] A. Beltratti and C. Morana: Statistical Benefits of Value-at-risk with long memory. Journal of Risk 7(2005), p. 21-45.

[5] A.-M. Fuertes and J. Olmo: Optimally Harnessing Inter-Day and Intra-Day Information for Daily Value-at-Risk Prediction. International Journal of Forecast 29(2013), p. 28-42.

[6] F. Corsi: A Simple Approximate Long-Memory Model of Realized Volatility. Journal of Financial Econometrics 7, 2 (2009), p. 174-196.

[7] P. Giot and S. Laurent: Modelling Daily Value-at-Risk Using Realized Volatility and ARCH Type Models. Journal of Empirical Finance 11 (2004), p. 379-398.

[8] T. Klein: Conditional Variance Dynamics of Gold and Other Precious Metals Forecast Comparison with Intra-Day Data. (2016) Preprint submitted to Elsevier. https://ssrn.com/ abstract $=2815326$.

[9] N. Todorova, A. Worthington, and M. Souček: Realized Volatility Spillovers in the Non-Ferrous Metal Futures Market. Resources Policy 39 (2014), p. 21-31.

[10]T.G. Andersen, D. Dobrev, E. Schaumburg: Jump-Robust Volatility Estimation using Nearest Neighbor Truncation. NBER Working Paper No. 15533. (2010)

[11]H.E. Hurst: Long-term Storage Capacity of Reservoirs. Transactions of the American Society of Civil Engineers 116 (1951), p. 770-799.

[12] J. Geweke, and S. Porter-Hudak: The Estimation and Application of Long Memory Time Series Models, Journal of Time Series Analysis 4 (1983), 221-238.

[13]M. Sarma, S. Thomas, and A. Shah: Selection of VaR Models. Journal of Forecasting 22(4) (2003), p. 337-358. 